\title{
Charge order, enhanced orbital moment, and absence of magnetic frustration in layered multiferroic $\mathrm{LuFe}_{2} \mathrm{O}_{4}$
}

\author{
K. Kuepper, ${ }^{1, *}$ M. Raekers, ${ }^{2, \dagger}$ C. Taubitz,${ }^{2}$ M. Prinz,${ }^{2}$ C. Derks, ${ }^{2}$ M. Neumann,,${ }^{2,}$ A. V. Postnikov, ${ }^{3}$ F. M. F. de Groot, ${ }^{4}$ \\ C. Piamonteze, ${ }^{5}$ D. Prabhakaran, ${ }^{6}$ and S. J. Blundell ${ }^{6}$ \\ ${ }^{1}$ Institute of Ion Beam Physics and Materials Research, Forschungszentrum Dresden-Rossendorf, \\ P.O. Box 5101 19, D-01314 Dresden, Germany \\ ${ }^{2}$ Department of Physics, University of Osnabrück, D-49069 Osnabrück, Germany \\ ${ }^{3}$ LPMD, Institute Jean Barriol and Paul Verlaine University, Metz, France \\ ${ }^{4}$ Department of Inorganic Chemistry and Catalysis, Utrecht University, Sorbonnelaan 16, 3584 CA Utrecht, Netherlands \\ ${ }^{5}$ Swiss Light Source, Paul Scherrer Institut, 5232 Villigen PSI, Switzerland \\ ${ }^{6}$ Department of Physics, Clarendon Laboratory, University of Oxford, Parks Road, Oxford OX1 3PU, United Kingdom
}

(Received 13 November 2009; published 18 December 2009)

\begin{abstract}
Electronic and magnetic properties of the charge ordered phase of $\mathrm{LuFe}_{2} \mathrm{O}_{4}$ are investigated by means of $\mathrm{x}$-ray spectroscopic and theoretical electronic structure approaches. $\mathrm{LuFe}_{2} \mathrm{O}_{4}$ is a compound showing fascinating magnetoelectric coupling via charge ordering. Here, we identify the spin ground state of $\mathrm{LuFe}_{2} \mathrm{O}_{4}$ in the charge ordered phase to be a 2:1 ferrimagnetic configuration, ruling out a frustrated magnetic state. An enhanced orbital moment may enhance the magnetoelectric coupling. Furthermore, we determine the densities of states and the corresponding correlation potentials by means of x-ray photoelectron and emission spectroscopies, as well as electronic structure calculations.
\end{abstract}

DOI: $10.1103 /$ PhysRevB.80.220409

PACS number(s): 75.80.+q, 71.20.-b, 78.70.Dm, 78.70.En

Multiferroic transition metal oxides, i.e., compounds in which more than one ferroic phase coexist, have gained enormous attention during the last few years. ${ }^{1-4}$ Besides a number of perovskites and related compounds, ${ }^{2,5,6}$ the charge frustrated layered compound $\mathrm{LuFe}_{2} \mathrm{O}_{4}$ has attracted intense interest due to its fascinating ferroelectric and magnetoelectric properties. ${ }^{7,8} \mathrm{LuFe}_{2} \mathrm{O}_{4}$ has a rhombohedral crystal structure (space group $R \overline{3} \mathrm{~m}$ ). The underlying layered structure consists of W-like hexagonal $\mathrm{Fe}_{2} \mathrm{O}_{2.5}$ and U-like $\mathrm{LuO}_{1.5}$ layers. ${ }^{9}$ The $\mathrm{W}$ layers comprise two triangular nets of $\mathrm{Fe}$ ions; the resulting electric polarization is induced via a frustrated charge ordering of $\mathrm{Fe}^{2+}$ and $\mathrm{Fe}^{3+}$ ions on the resulting honeycomb lattice below $330 \mathrm{~K}^{10-12}$ Below $240 \mathrm{~K}$ a longrange ferrimagnetic order sets in. ${ }^{7}$ The fact that the ferroelectricity is caused by correlated electrons from the $\mathrm{Fe}$ ions leads to unusual properties and unique capabilities of $\mathrm{LuFe}_{2} \mathrm{O}_{4}$. A large response of the dielectric constant by applying small magnetic fields has been found, opening a possible route for future devices. ${ }^{8}$ Phase transitions from the charge ordered $(\mathrm{CO})$ phase have been very recently associated with a nonlinear current-voltage behavior and an electric-field-induced phase transition, which might be of interest for potential electric-pulse-induced resistive switching applications. ${ }^{13,14}$

The large magnetoelectric coupling has been attributed to an intricate interplay between charge and spin degrees of freedom with the crystal lattice and external electrical and magnetic fields to some extent on a short-range order. ${ }^{15-19}$ However, there is still some confusion about the nature of spin-charge coupling in $\mathrm{LuFe}_{2} \mathrm{O}_{4}$. In particular a model finding a $\sqrt{3} \times \sqrt{3} \mathrm{CO}$ ground state ${ }^{20,21}$ is challenged by simulations implying that the electrical polarization in $\mathrm{LuFe}_{2} \mathrm{O}_{4}$ is due to spin-charge coupling and a spin frustrated magnetic ground state in a chain $\mathrm{CO}$ state. ${ }^{22,23}$ On the other hand the first model finds a ferrimagnetic spin ground state where
$\mathrm{Fe}^{2+}$ and $1 / 3$ of $\mathrm{Fe}^{3+}$ make up the majority spin, and 2/3 of $\mathrm{Fe}^{3+}$ make up the minority spin.

$\mathrm{X}$-ray magnetic circular dichroism (XMCD) is a very powerful tool to investigate the internal magnetic structure of the compound in question, including the unique possibility to separate the magnetic moments into their spin and orbital contributions. We applied XMCD to the Fe $L$ edge in the charge ordered phase around $270 \mathrm{~K}$ and compare these results to corresponding full multiplet calculations. Furthermore, we performed x-ray photoelectron spectroscopy (XPS) and $\mathrm{x}$-ray emission spectroscopy (XES) studies of the valence band. These results are compared to first-principles band-structure calculations in order to determine the Coulomb repulsion correlation strength between the Fe $3 d$ and $\mathrm{Lu} 4 f$ ions in $\mathrm{LuFe}_{2} \mathrm{O}_{4}$. High-quality single crystals were grown using a four-mirror floating zone furnace, as described elsewhere. $^{24}$

Core levels and valence band were recorded by means of XPS, using a PHI5600ci multitechnique spectrometer with monochromatic Al $K \alpha$ radiation with an overall resolution of about $0.3 \mathrm{eV}$. The XMCD, XAS, and XES measurements were performed at beamlines 4.0.2 and 8.0.1 at the Advanced Light Source in Berkeley, California. In order to get a sample surface free of contamination the crystal was cleaved in situ just prior to the XPS and XMCD experiments. In case of the bulk sensitive XES the sample was rinsed with isopropanol before mounting the sample into the transfer chamber. The $a b$ initio electronic structure calculations were performed using the (linearized) full-potential augmented plane-wave method $^{25}$ of the density-functional theory, as implemented in the WIENK2K code. ${ }^{26}$ The Fe $L_{2,3}$ XAS and XMCD are compared to full multiplet simulations which were carried out with the $T T$-multiplets program package. ${ }^{27,28}$

Figure 1 displays the $\mathrm{Fe} 2 p$ XPS of $\mathrm{LuFe}_{2} \mathrm{O}_{4}$ along with the spectra of reference compounds, namely, $\mathrm{FeO}\left(\mathrm{Fe}^{2+}\right)$, 


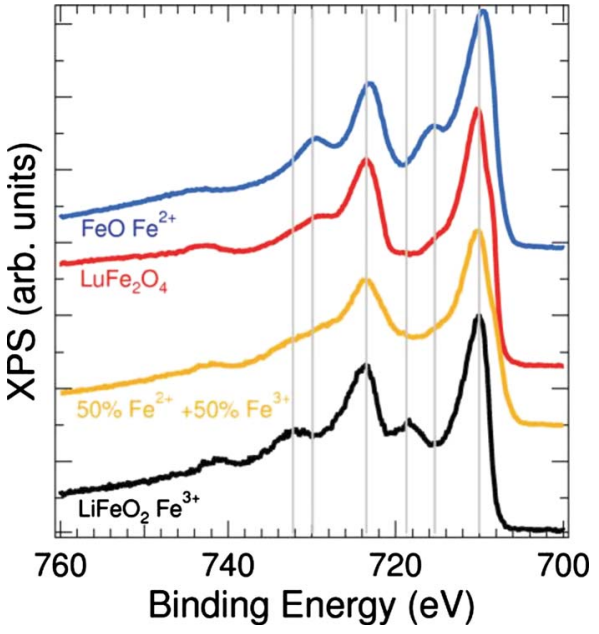

FIG. 1. (Color online) Fe $2 p$ XPS of $\mathrm{LuFe}_{2} \mathrm{O}_{4}, \mathrm{FeO}\left(\mathrm{Fe}^{2+}\right.$ reference), and $\mathrm{LiFeO}_{2}\left(\mathrm{Fe}^{3+}\right.$ reference).

$\mathrm{LiFeO}_{2}\left(\mathrm{Fe}^{3+}\right)$, and a $1: 1$ superposition of both reference spectra, representing a result for a nominally $\mathrm{Fe}^{2.5+}$ based oxide since XPS is significantly more sensitive to the electronic configuration (charge transfer) than to the local symmetry. All spectra were recorded directly after cleaving the samples in ultrahigh vacuum in order to avoid any surface contamination. The Fe $2 p_{3 / 2}$ peak of $\mathrm{LuFe}_{2} \mathrm{O}_{4}$ at $710 \mathrm{eV}$ has a shoulder at $709 \mathrm{eV}$, which is due to the mixed Fe valence state in this compound. The $\mathrm{Fe} 2 p_{1 / 2}$ peak is located at 705 $\mathrm{eV}$. From the chemical shift of the Fe main peaks one cannot easily determine the valence state of $\mathrm{Fe}$, following the usual procedure, because of the smallness of the shift. The satellites give better information about the valence state of the $\mathrm{Fe}$ ions. In the $\mathrm{Fe}^{2+}$ reference sample two satellites are present at 716 and $730 \mathrm{eV}$. The satellites of the $\mathrm{Fe}^{3+}$ reference are located at 718 and $732 \mathrm{eV}$. The $\mathrm{LuFe}_{2} \mathrm{O}_{4} \mathrm{Fe} 2 p$ spectrum shows clearly the presence of both types of satellites, yielding in fact a very good agreement with the superimposed $\left(0.5 \mathrm{Fe}^{2+}+0.5 \mathrm{Fe}^{3+}\right)$ reference spectrum.

The electronic structure of occupied states was investigated by XPS of the valence band and XES from $\mathrm{Fe} 3 d \rightarrow \mathrm{Fe} 2 p$ and $\mathrm{O} 2 p \rightarrow \mathrm{O} 1 s$. The results are presented in Fig. 2 in comparison with GGA $+U$ calculations, in which the $U$ value of 0.7 Ry was selected for $\mathrm{Lu} 4 f$ and $U=0.3 \mathrm{Ry}$ for $\mathrm{Fe} 3 d$, guided by the best agreement with the valence-band XPS in the placement of respective peaks. In the XPS spectrum two peaks of the Lu $4 f$ multiplet are located at -7.5 and $-9 \mathrm{eV}$. The structure from -3 to $-6 \mathrm{eV}$ is distributed to $\mathrm{O} 2 p$ states hybridized with $\mathrm{Fe} 3 d$ states, which is in comparison with the XES spectra of the $\mathrm{Fe} L$ and the $\mathrm{O} K$ edges. The small states close to the Fermi level (from 0 to $-1 \mathrm{eV}$ ) can be interpreted as highest occupied Fe $3 d\left(d_{x y}, d_{x^{2}-y^{2}}\right)$ states of the divalent sites in comparison with band-structure calculations of Xiang and Whangbo. ${ }^{20}$

The band-structure calculations presented in Fig. 2 are in excellent agreement with the XES and XPS measurements. It should be noted that the present calculation ignores charge ordering and assumes a minimal single unit cell of seven atoms. We checked that, in agreement with earlier calculations, a tripled unit cell with different magnetic and charge

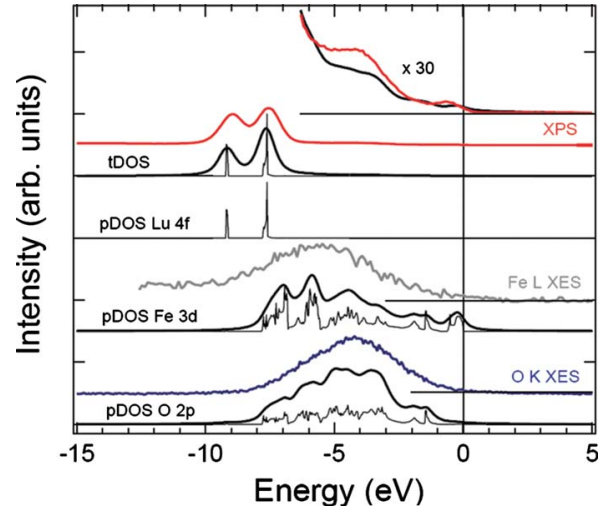

FIG. 2. (Color online) XPS valence band, Fe $L$ edge, and O $K$ edge XES in comparison with $\mathrm{GGA}+U$ calculations.

states over six Fe sites results in the opening of a band gap. However, a meaningful identification of all important features in the electronic structure is already possible on the basis of the single-cell calculation. The states close to the Fermi level in the XPS valence band can be identified as highest occupied Fe $3 d$ states. Remarkable agreement is obtained between the XPS and the calculation in the range from $-5 \mathrm{eV}$ to the Fermi level as can be seen in the 30 times enlarged spectra in the top of Fig. 2. The strong hybridization between $\mathrm{Fe} 3 d$ and $\mathrm{O} 2 p$ becomes clear if one compares the peak structures of the calculated density of states of these states where the most intense $\mathrm{Fe} 3 d$ states are located at -6 and $-7 \mathrm{eV}$ and the most intense $\mathrm{O} 2 p$ states are located at -3.5 and $-4.5 \mathrm{eV}$. Here, it is very clear that the states at higher binding energy $(\sim-6 \mathrm{eV})$ have a $d$ character and the states at $\sim-4 \mathrm{eV}$ have a $p$ character, as was found in Ref. 29 for $\mathrm{KTaO}_{3}$ and $\mathrm{KNbO}_{3}$. For two pronounced peaks identified with $\mathrm{Fe} 3 d$ states at -7 and $-6 \mathrm{eV}$, the lower is primarily formed by $d_{z^{2}}$ and $d_{x y}+d_{x^{2}-y^{2}}$ states, of which the latter do equally contribute to the peak at $-6 \mathrm{eV}$. This upper peak is otherwise built by the $d_{x z}+d_{y z}$ states. This agrees with the results of Xiang et al. In our calculation, the hybridization between $\mathrm{Fe} 3 d$ and $\mathrm{O} 2 p$ states seems slightly stronger than in previously published works; this strength must to some extent depend on the $U$ value used. Note that the above labeling of the $3 d$ orbitals is only approximative in the structure in question, as the spin and orbital diversification and related distortions would reduce the symmetry. The results give rise to different combinations of $d$ orbitals-see Khomskii and Kugel. ${ }^{30}$

The hybridization between $\mathrm{Fe} 3 d$ and $\mathrm{O} 2 p$ states, a priori important for holding together the $\mathrm{W}$ layer, does not result in a substantial delocalization of magnetic moments, thus justifying our single-ion multiplet calculations. It can be concluded that magnetic density from $\mathrm{Fe}$ sites is only slightly spilled onto $\mathrm{O}$ in the $\mathrm{W}$ layer, not onto (equally close) $\mathrm{O}$ in the $\mathrm{U}$ layer. Note that this effect must be in fact somehow overestimated in the present calculation because of the ferromagnetic structure assumed, and in the genuine spincharge mixed structure the localization of magnetic moments on the Fe sites should be stronger.

In Fig. 3 the XMCD spectra of the Fe $L$ edge, measured in total electron yield (TEY) mode at $\sim 150 \mathrm{~K}$, are presented in 


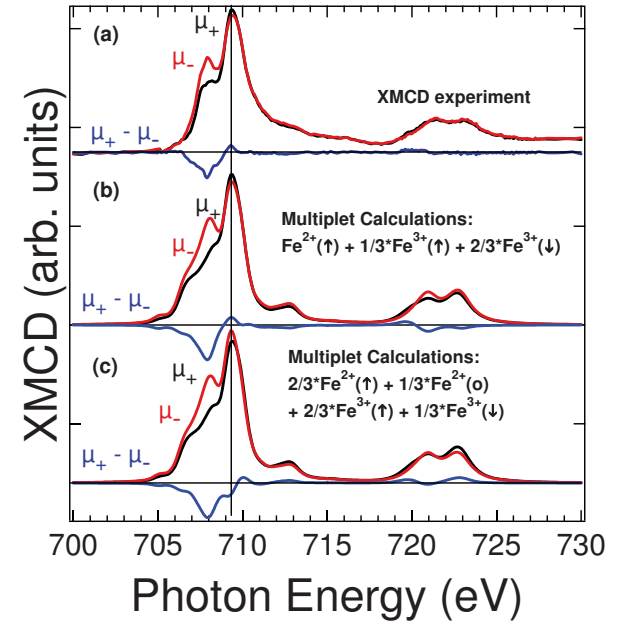

FIG. 3. (Color online) (a) Fe $L$ edge XMCD performed at 150 $\mathrm{K}$, and (b) and (c) ligand field multiplet simulations considering different possible spin orderings.

the upper part of the graph, with ligand multiplet calculations in $C_{3 i}$ symmetry plotted in the lower part of the graph for comparison. The sample was cleaved at an ambient pressure of around $5 \times 10^{-5} \mathrm{mbar}$ and a temperature of around $80 \mathrm{~K}$ (nitrogen precooling) before being transferred into the helium cryostat with a pressure of around $5 \times 10^{-8} \mathrm{mbar}$. The $c$ axis of the $\mathrm{LuFe}_{2} \mathrm{O}_{4}$ single crystal was aligned parallel to the external applied magnetic field $(6 \mathrm{~T})$.

The $\mathrm{Fe}^{2+}$ and $\mathrm{Fe}^{3+}$ peaks of the $L_{3}$ edge are located at 708 and $709.5 \mathrm{eV}$, respectively. The $\mathrm{Fe}^{3+}$ contribution is somewhat higher than $50 \%$ (due to the relatively high ambient pressure during cleaving the sample), and we had to take into account an additional $\mathrm{Fe}^{3+}$ contribution in order to get the best fit to the XAS. A comparison of fluorescence yield to electron yield XAS measurements, not shown here, revealed a contribution of the additional $\mathrm{Fe}^{3+}$ signal in the TEY spectra of about 50\% compared to the bulk signal. This signal has been assumed not to contribute to the XMCD signal. The Fe $L_{2}$-edge peaks are located at 721 and $723 \mathrm{eV}$ with a shoulder at $720 \mathrm{eV}$. The experimental dichroic signal at the $L_{2}$ edge is very small, but the calculated dichroism at the $L_{2}$ edge is in agreement with experiment. The $L_{3}$ edge shows a clear dichroism at the $\mathrm{Fe}^{2+}$ peak, whereas a smaller dichroism at $\mathrm{Fe}^{3+}$ is of the opposite sign. This is a clear indication of all $\mathrm{Fe}^{2+}$ sites being in the majority-spin state, while $1 / 3$ of the $\mathrm{Fe}^{3+}$ spin being in majority and $2 / 3$ in minority. This configuration is also used for the multiplet calculations shown in Fig. 3(b) and gives a very good agreement with the experiments besides the shoulder at $707 \mathrm{eV}$, which is overestimated by the calculation. In particular the reversal sign in the XMCD between 708 and $709.5 \mathrm{eV}$ visible in the experimental data is perfectly reproduced by the simulations. We also calculated a frustrated spin configuration according to Nagano et al. ${ }^{22}$ The results are shown in Fig. 3(c). As one can see there are significant differences between the measured spectra and the simulations, indicating that the $\mathrm{Fe}$ ions are not in a spin frustrated ground state.

This nonfrustrated spin configuration results in a saturation magnetic moment of the spin moment of $4 \mu_{B}-(2 / 3) 5 \mu_{B}+(1 / 3) 5 \mu_{B}=2.33 \mu_{B}$ /f.u., considering a purely ionic configuration. The maximal orbital moment for $\mathrm{Fe}^{2+}$ in a trigonal bipyramidal crystal field and in high spin state is $m_{o r b}= \pm 2 \mu_{B} /$ f.u. for the occupation of $d_{x y}$ or $d_{x^{2}-y^{2}}$. $\mathrm{Fe}^{3+}$ in high spin state has no orbital moment due to completely filled spin-up states and completely empty spin-down states. This results in a maximal total magnetic moment of $m_{\text {tot }}=2.33 \mu_{B} /$ f.u. $+2 \mu_{B} /$ f.u. $=4.33 \mu_{B}$ /f.u. In our experiment the spin and orbital sum rules give a spin magnetic moment equal to $1.1 \mu_{B} /$ f.u. and an orbital moment of $0.76 \mu_{B} /$ f.u. The simulated spectra and the expectation values obtained from the multiplet program were used to calculate a correction factor of 0.55 for the spin sum rule. The simulated spectra and the expectation values obtained from the multiplet program were used to calculate a correction factor to the effective spin sum rule. The correction factor found is 0.55 , which also corrects for the non-negligible value of the magnetic dipole term $\left(T_{z}\right)$ found to be 0.1 from the $\mathrm{Fe}^{2+}$ simulations. The relatively high orbital moment can be explained by the anisotropy induced by the bipyramidal local environment, and it can be also determined from the multiplet calculation of $\mathrm{Fe}^{2+}$. The orbital moment is the reason for the spin-lattice interaction, and the magnetic easy axis is set by the orbital moment, which is fixed in the lattice by the electron configuration. Thus, this result clearly supports the model finding a $\sqrt{3} \times \sqrt{3} \mathrm{CO}$ ground state including significant intersheet spin-exchange interactions. ${ }^{20,21}$

In conclusion, we performed an electronic structure investigation of the charge frustrated multiferroic $\mathrm{LuFe}_{2} \mathrm{O}_{4}$ with special emphasis on the charge ordered phase at room temperature, leading to a detailed picture of the electronic and magnetic properties. We identified the electronic states of the valence band by means of valence-band XPS and XES, and $\mathrm{GGA}+U$ first-principles calculations. Fe $L$-edge XMCD and multiplet simulations demonstrate a strong coupling between the charge order and the magnetic structure of $\mathrm{LuFe}_{2} \mathrm{O}_{4}$. Furthermore, the quite large orbital moment found may enhance the magnetoelectric coupling.

Part of this work was performed at the Advanced Light Source (ALS), Lawrence Berkeley National Laboratory, Berkeley, USA, which is operated under Contract No. DEAC03-76SF00098. M.R. gratefully acknowledges financial support of the GRK695: Nonlinearities of optical materials. C.T. and M.P. were supportedd by the Lichtenberg program of the Federal State of Lower Saxony. 
*Present address: University of Ulm, Institut für Festkörperphysik, Albert-Einstein-Allee 11, D-89069 Ulm, Germany; karsten.kuepper@uni-ulm.de

†mraekers@uos.de

†mneumann@uos.de

${ }^{1}$ N. A. Hill, J. Phys. Chem. B 104, 6694 (2000).

${ }^{2}$ J. Wang et al., Science 299, 1719 (2003).

${ }^{3}$ M. Fiebig, J. Phys. D: Appl. Phys. 38, R123 (2005).

${ }^{4}$ S.-W. Cheong and M. Mostovoy, Nature Mater. 6, 13 (2007).

${ }^{5}$ N. Hur, S. Park, P. A. Sharma, J. S. Ahn, S. Guha, and S.-W. Cheong, Nature (London) 429, 392 (2004).

${ }^{6}$ M. Sakai, A. Masuno, D. Kan, M. Hashisaka, K. Takata, M. Azuma, M. Takano, and Y. Shimakawa, Appl. Phys. Lett. 90, 072903 (2007).

${ }^{7}$ N. Ikeda et al., Nature (London) 436, 1136 (2005).

${ }^{8}$ M. A. Subramanian, T. He, J. Chen, N. S. Rogado, T. G. Calvarese, and A. W. Sleight, Adv. Mater. 18, 1737 (2006).

${ }^{9}$ J. Kim, S. B. Kim, C. U. Jung, and B. W. Lee, IEEE Trans. Magn. 45, 2608 (2009).

${ }^{10}$ M. Isobe et al., Acta Crystallogr., Sect. C: Cryst. Struct. Commun. 46, 1917 (1990).

${ }^{11}$ Y. Yamada, K. Kitsuda, S. Nohdo, and N. Ikeda, Phys. Rev. B 62, 12167 (2000).

${ }^{12}$ N. Ikeda et al., Ferroelectrics 314, 41 (2005).

${ }^{13}$ L. J. Zeng, H. X. Yang, Y. Zhang, H. F. Tian, C. Ma, Y. B. Qin, Y. G. Zhao, and J. Q. Li, EPL 84, 57011 (2008).

${ }^{14}$ C. Li, X. Zhang, Z. Cheng, and Y. Sun, Appl. Phys. Lett. 93, 152103 (2008).

${ }^{15}$ M. Angst et al., Phys. Rev. Lett. 101, 227601 (2008).

${ }^{16}$ X. S. Xu et al., Phys. Rev. Lett. 101, 227602 (2008).
${ }^{17}$ W. Wu et al., Phys. Rev. Lett. 101, 137203 (2008).

${ }^{18}$ C.-H. Li, F. Wang, Y. Liu, X.-Q. Zhang, Z.-H. Cheng, and Y. Sun, Phys. Rev. B 79, 172412 (2009).

${ }^{19}$ J. Wen, G. Xu, G. Gu, and S. M. Shapiro, Phys. Rev. B 80, 020403(R) (2009).

${ }^{20}$ H. J. Xiang and M.-H. Whangbo, Phys. Rev. Lett. 98, 246403 (2007).

${ }^{21}$ H. Xiang, E. Kan, S. Wei, M. Whangbo, and J. Yang, Phys. Rev. B 80, 132408 (2009).

${ }^{22}$ A. Nagano, M. Naka, J. Nasu, and S. Ishihara, Phys. Rev. Lett. 99, 217202 (2007).

${ }^{23}$ M. Naka, A. Nagano, and S. Ishihara, Phys. Rev. B 77, 224441 (2008).

${ }^{24}$ J. Iida, S. Takekawa, and N. Kimizuka, J. Cryst. Growth 102, 398 (1990).

${ }^{25}$ D. J. Singh, Planewaves, Pseudopotentials and the LAPW Method (Kluwer Academic Publishers, Boston, 1994), p. 115.

${ }^{26}$ P. Blaha, K. Schwarz, G. K. H. Madsen, J. Kvasnicka, and D. Luitz, An Augmented Plane Wave + Local Orbitals Program for Calculating Crystal Properties (Karlheinz Schwarz, Techn. Universität Wien, Wien, Austria, 2001).

${ }^{27}$ F. M. F. de Groot, J. Electron Spectrosc. Relat. Phenom. 62, 111 (1993).

${ }^{28}$ F. M. F. de Groot and A. Kotani, Core Level Spectroscopy of Solids, Advances in Condensed Matter Science (CRC Press, Boca Raton, FL, 2008).

${ }^{29}$ A. Winiarski, T. Neumann, B. Mayer, G. Borstel, and M. Neumann, Phys. Status Solidi B 183, 475 (1994).

${ }^{30}$ D. I. Khomskii and K. I. Kugel, Phys. Rev. B 67, 134401 (2003). 